\title{
Influencia de las variables sociodemográficas y socioeducativas en el rendimiento académico de alumnos del Grado en Fisioterapia
}

\author{
Mercedes Soto-González, Iria Da Cuña-Carrera, Eva M. Lantarón-Caeiro, M. Teresa Labajos-Manzanares
}

Objetivo. Conocer la influencia de las variables sociodemográficas y socioeducativas sobre el rendimiento académico de los alumnos del Grado en Fisioterapia.

Sujetos y métodos. La muestra estaba formada por 151 alumnos matriculados en el Grado en Fisioterapia de la Universidad de Vigo en el curso 2010-2011. Mediante una encuesta se obtuvieron los datos necesarios relativos a las variables sociodemográficas y socioeducativas; para medir el rendimiento académico se tuvo en cuenta, además de la nota media, la tasa de rendimiento, la tasa de intento y la tasa de éxito. Se aplicaron contrastes estadísticos bivariantes para la correlación de dichas variables.

Resultados. Variables sociodemográficas como la edad, la residencia, el compaginar estudios y trabajo o tener otras obligaciones, además de los estudios, influyen sobre el rendimiento académico. En cuanto a las variables socioeducativas, se ha comprobado que el curso, la vía de ingreso, la primera opción de matrícula o ser repetidor son variables que se correlacionan con el rendimiento académico.

Conclusiones. Existen numerosas variables que afectan al rendimiento académico, pero tan sólo la residencia durante el curso, el curso académico y la vía de ingreso a los estudios afectan finalmente a la nota media.

Palabras clave. Educación superior. Estudiantes. Fisioterapia. Rendimiento académico.

Influence of socio-demographic-educational variables on the academic performance of students of the Physical Therapy Degree

Aim. To determine the influence of socio-demographic and socio-educational variables on the academic performance of students of the Physical Therapy Degree.

Subjects and methods. The population was comprised of 151 students enrolled in the Physical Therapy degree. Through a survey, we have obtained the necessary data on socio-demographic and socio-educational variables. In order to measure the academic performance, it has been taken into account, in addition to the grade point average, the rate of return, the rate of attempts and the success rate.

Results. Socio-demographic variables such as age, residence, combining studies and work or having other obligations in addition to the studies, impact on academic performance in terms of socio-educational variables. We have found out that the course, the route of entry, the first enrollment option or being a student repeating a year are variables that correlate with academic performance.

Conclusions. There are many variables that affect academic performance, however, only the residence during the course, the academic course and the entrance road to the studies ultimately affect the average mark.

Key words. Academic performance. Higher education. Physical therapy. Students.

\section{Introducción}

El rendimiento académico es un concepto complejo y, por ello, no está consensuado qué se entiende como tal. En lo que sí coinciden los diferentes estudios es que se trata de un ente multifactorial de di- fícil medida. En general, se reconocen determinantes personales, sociales e institucionales en el rendimiento [1]. El rendimiento académico se podría definir como una medida de las capacidades correspondientes o indicativas que manifiesta, de manera estimada, aquello que una persona ha aprendido
Departamento de Biología Funcional y Ciencias de la Salud; Facultad de Fisioterapia; Universidad de Vigo; Pontevedra (M. Soto-González, I. Da Cuña-Carrera, E.M LantarónCaeiro). Departamento de Psiquiatría y Fisioterapia; Facultad de Ciencias de la Salud; Universidad de Málaga; Málaga, España (M.T. LabajosManzanares).

\section{Correspondencia:}

Iria Da Cuña Carrera. Facultad de Fisioterapia. Universidad de Vigo. Campus a Xunqueira, s/n. E-36001 Pontevedra.

E-mail:

iriadc@uvigo.es

Conflicto de intereses: No declarado.

Competing interests: None declared.

(c) $2015 \mathrm{FEM}$ 
como consecuencia de un proceso de instrucción o formación [2].

En los estudiantes universitarios constituye un factor imprescindible y fundamental para la valoración de la calidad educativa en la enseñanza superior [3], siendo la resultante del complejo mundo que envuelve al estudiante, determinado por una serie de aspectos cotidianos (esfuerzo, capacidad de trabajo, intensidad de estudio, competencias, aptitud, personalidad, atención, motivación, memoria, medio relacional) que afectan directamente el desempeño académico de los individuos [4]. Por tanto, se considera la suma de diferentes y complejos factores que actúan en la persona que aprende y se ha definido con un valor atribuido al logro del estudiante en las tareas académicas.

Con la implantación y el proceso de desarrollo del Espacio Europeo de Educación Superior, las universidades, para contribuir a la calidad, deben rendir cuentas a la sociedad midiendo los resultados del proceso formativo que desarrollan. Estos resultados han de incluirse en las memorias de verificación y de seguimiento de la titulación, en términos de tasas de eficiencia, rendimiento o abandono [5].

Caso y Hernández, en un estudio de adolescentes, determinaron la existencia de ciertas variables que afectaban al rendimiento desde los niveles académicos previos a la universidad y manifestaban que 'el contar con datos que arrojen luz sobre el posible efecto de otras variables de índole personal sobre el rendimiento escolar, permitiría el diseño e instrumentación de programas preventivos dirigidos a la población general en riesgo de fracaso escolar, debido a su exposición al efecto nocivo de variables distales como el nivel socioeconómico o la escolaridad de los padres' [6].

Ya en el ámbito universitario, conocer los diferentes factores que inciden en el rendimiento académico en el campo de la educación superior de una manera más integral permite obtener resultados tanto cualitativos como cuantitativos para propiciar un enfoque más completo en la toma de decisiones para mejorar los niveles de pertinencia, equidad y calidad educativa [7].

De entre los factores que influyen en el rendimiento, los recursos familiares, su nivel de estudios, los hábitos de trabajo, la orientación y el apoyo académico, las actividades culturales que se realizan, los libros que se leen, la estimulación para explorar y discutir ideas y acontecimientos y las expectativas sobre el nivel de estudios que pueden alcanzar los hijos, son factores que tienen una influencia muy importante en la educación de éstos [8]. Procesos que vinculan a la familia y al desempeño académico son el intercambio verbal entre la madre y los hijos, las expectativas familiares acerca del desempeño académico, las relaciones positivas entre padres e hijos, las creencias de los padres acerca de sus hijos, y las atribuciones que hacen al comportamiento de éstos y las estrategias de control y disciplina [9].

Las investigaciones dirigidas al estudio de los factores que inciden en el rendimiento académico de los estudiantes universitarios son numerosas e incorporan una amplia variedad de posibles determinantes. Desde los factores de carácter personal (sexo, edad, situación sociofamiliar, aptitudes intelectuales...), pasando por los factores académicos (estudios realizados, rendimientos previos...), hasta los determinantes de tipo pedagógico (metodologías educativas, sistemas de evaluación...) [10].

El objetivo de este estudio es conocer la influencia de las variables sociodemográficas y socioeducativas sobre el rendimiento académico de los alumnos del Grado en Fisioterapia.

\section{Sujetos y métodos}

Se ha realizado un estudio descriptivo transversal utilizando como instrumento de recogida de datos un cuestionario en formato papel.

\section{Muestra}

El estudio se realizó sobre los alumnos de Grado en Fisioterapia de la Universidad de Vigo, campus de Pontevedra. La población total estaba compuesta por 188 alumnos, de los cuales 151 aceptaron participar voluntariamente $(80,31 \%)$. Del total de la muestra, 105 sujetos eran mujeres y 46 eran hombres, con edades comprendidas entre 18 y 46 años, siendo la edad media de 21,67 años, y la mediana, de 21.

\section{Instrumentos}

Mediante un cuestionario se obtuvieron los datos necesarios relativos a las variables sociodemográficas y socioeducativas. Se trata de un cuestionario con preguntas sencillas y con una respuesta que en la mayor parte de los casos es afirmativa o negativa.

Las variables sociodemográficas analizadas fueron la edad, el sexo, la residencia durante el curso, si poseían o no otras obligaciones además de los estudios, y si trabajaban en la actualidad. Por su parte, las variables socioeducativas estudiadas fueron el curso, la vía de ingreso a la universidad, la nota de acceso, los estudios del padre, los estudios de la madre y la condición de ser repetidor. 
Para la medición del rendimiento académico se utilizó, además de la nota media, la tasa de rendimiento (relación porcentual entre el número de créditos superados por el alumno y el número de créditos en los que se había matriculado), la tasa de intento (relación porcentual entre el número de créditos presentados a examen y el número de créditos en los que se había matriculado) y la tasa de éxito (relación porcentual entre el número de créditos superados y el número de créditos presentados a examen), ya que se consideró que la nota media, por sí misma, no era objetiva para medir el rendimiento del alumno: un alumno puede tener una nota media mayor que otro, pero estar matriculado en un número muy inferior de créditos.

\section{Procedimiento}

El cuestionario fue cumplimentado por los alumnos en horas de clase de materias de formación básica u obligatoria. Se introdujeron los datos en una base de datos y se cruzaron con los expedientes académicos de los alumnos, los cuales autorizaron de forma explícita, mediante un consentimiento informado, la utilización de sus datos, ya que las respuestas del cuestionario no eran anónimas. Para el análisis estadístico se empleó el programa SPSS v. 15.0. Las pruebas estadísticas utilizadas para el análisis fueron la $t$ de Student, la prueba paramétrica ANOVA y la prueba post-hoc de Scheffé, estableciéndose la significación cuando $\mathrm{p} \leq 0,05$.

\section{Resultados}

En cuanto al análisis descriptivo de las variables sociodemográficas, el $57 \%$ residía con su familia durante el curso académico. El 17,9\% tenía otras obligaciones además de los estudios. En lo referente a su vida laboral, el 21,2\% compaginaba estudios y trabajo.

Respecto a las variables socioeducativas, la vía de acceso fue mayoritariamente la selectividad, con un $75,5 \%$ de los alumnos; el $22,5 \%$ procedían de la formación profesional, el 1,3\% eran deportistas de alto nivel y el $0,7 \%$ accedían por la prueba de acceso a mayores de 25 años. El 63,6\% del alumnado se matriculó en fisioterapia como primera opción y el $24,5 \%$ era repetidor de alguna de las materias.

En la tabla I se muestra la media y la desviación estándar de los indicadores de rendimiento académico según las variables sociodemográficas y socioeducativas estudiadas.

Para analizar la relación entre el rendimiento académico y la edad, se dividió la muestra en dos gru- pos: mayores y menores de 25 años. Los resultados del estudio indican que la edad se relaciona significativamente con la nota media, la tasa de rendimiento y la tasa de éxito, de forma que el alumnado mayor de 25 años puntuaba por debajo tanto en la nota media $(p=0,02)$ como en las tasas de rendimiento $(p=0,02)$ y éxito $(p=0,03)$.

En relación al sexo, la tendencia es que las mujeres poseían valores ligeramente más altos que los hombres, pero al realizar la comparación de medias mediante la prueba $t$ de Student se obtuvo que la relación entre estas variables no era estadísticamente significativa $(p=0,65)$.

Los alumnos que residían con su familia tenían una nota media mayor que los que no vivían con sus padres $(p=0,06)$; en las tasas de rendimiento, intento y éxito no se apreciaban diferencias significativas $(p>0,05)$.

Los alumnos que trabajaban aprobaban menos y necesitaban más tiempo para finalizar sus estudios, pero no por ello obtenían una nota media peor. Las puntuaciones obtenidas en los indicadores de rendimiento académico en función de la situación laboral de los estudiantes se muestran en la tabla II.

Con respecto a los alumnos que tenían otras obligaciones, además de los estudios, cabe destacar que alcanzaban puntuaciones medias menores en los indicadores de rendimiento, pero no era estadísticamente significativo.

Para la vía de ingreso, como la muestra en los grupos de mayores de 25, deportistas de élite y mayores de 40 años u otra carrera era insuficiente, se consideraron solamente las variables selectividad y formación profesional, y por ello se realizó una comparación entre muestras independientes con la prueba $t$ de Student. Se observaron diferencias significativas entre los alumnos cuya vía de ingreso fue la selectividad con respecto a los de formación profesional, y se apreció que la nota media, la tasa de rendimiento y la tasa de éxito fueron mayores para los primeros (Tabla III).

Para efectuar el análisis de las variables rendimiento académico y nota de acceso, se segmentó el archivo de datos porque los alumnos de primer curso presentaban una nota de acceso diferente, ya que su cálculo se realizó sobre un baremo distinto, establecido en el Real Decreto 189/2008 por el cual se regulaban las condiciones para el acceso a las enseñanzas universitarias de grado y los procedimientos de admisión a las universidades públicas españolas [11]. En la tabla IV se observan las correlaciones entre la nota de acceso y el curso académico.

Atendiendo a la opción de matrícula, se comprueba que los alumnos que no habían elegido fisiotera- 
Tabla I. Indicadores de rendimiento académico en función de las variables sociodemográficas y socioeducativas (media \pm desviación estándar).

\begin{tabular}{|c|c|c|c|c|c|}
\hline & & Nota media & Tasa de rendimiento & Tasa de intento & Tasa de éxito \\
\hline \multirow{2}{*}{ Edad } & $<25$ años & $7,465 \pm 0,95$ & $88,846 \pm 19,06$ & $97,473 \pm 9,24$ & $90,746 \pm 16,59$ \\
\hline & $>25$ años & $6,849 \pm 1,05$ & $70,730 \pm 28,28$ & $92,292 \pm 12,22$ & $74,364 \pm 26,99$ \\
\hline \multirow{2}{*}{ Sexo } & Femenino & $7,49 \pm 1,00$ & $87,34 \pm 21,24$ & $97,78 \pm 6,175$ & $88,67 \pm 19,90$ \\
\hline & Masculino & $7,207 \pm 0,87$ & $86,36 \pm 19,86$ & $95,08 \pm 14,76$ & $90,13 \pm 14,70$ \\
\hline \multirow{2}{*}{$\begin{array}{l}\text { Residencia } \\
\text { con su familia }\end{array}$} & Sí & $7,53 \pm 0,84$ & $89,45 \pm 17,89$ & $98,06 \pm 5,33$ & $90,88 \pm 16,55$ \\
\hline & No & $7,22 \pm 1,10$ & $83,77 \pm 23,80$ & $95,49 \pm 13,31$ & $86,78 \pm 26,57$ \\
\hline \multirow{2}{*}{ Otras obligaciones } & Sí & $7,223 \pm 1,10$ & $77,948 \pm 23,80$ & $92,894 \pm 13,31$ & $83,57 \pm 20,57$ \\
\hline & No & $7,443 \pm 0,84$ & $89,027 \pm 17,89$ & $97,843 \pm 5,33$ & $90,32 \pm 16,55$ \\
\hline \multirow{2}{*}{ Trabaja } & Sí & $7,245 \pm 1,16$ & $77,172 \pm 24,94$ & $92,378 \pm 17,94$ & $82,984 \pm 20,83$ \\
\hline & No & $7,439 \pm 0,92$ & $89,421 \pm 18,95$ & $98,11 \pm 5,48$ & $90,5468 \pm 17,62$ \\
\hline \multirow{4}{*}{ Curso } & Primero & $6,69 \pm 0,94$ & $81,79 \pm 21,77$ & $97,69 \pm 6,47$ & $83,16 \pm 20,29$ \\
\hline & Segundo & $7,17 \pm 0,63$ & $87,97 \pm 22,60$ & $94,89 \pm 16,97$ & $91,77 \pm 16,59$ \\
\hline & Tercero & $7,37 \pm 0,79$ & $84,80 \pm 21,75$ & $97,61 \pm 6,44$ & $86,12 \pm 20,59$ \\
\hline & Cuarto & $8,31 \pm 0,66$ & $93,50 \pm 15,49$ & $97,36 \pm 6,44$ & $86,12 \pm 20,59$ \\
\hline \multirow{2}{*}{$\begin{array}{l}\text { Vía de ingreso } \\
\text { a la universidad }\end{array}$} & Selectividad & $7,645 \pm 0,86$ & $90,922 \pm 18,24$ & $97,527 \pm 9,74$ & $92,844 \pm 15,39$ \\
\hline & Formación profesional & $6,648 \pm 0,94$ & $74,613 \pm 23,73$ & $95,517 \pm 8,98$ & $76,885 \pm 22,63$ \\
\hline \multirow{3}{*}{ Estudios del padre } & Básicos & $7,46 \pm 1,02$ & $84,67 \pm 23,23$ & $98,07 \pm 5,34$ & $85,69 \pm 22,29$ \\
\hline & Medios & $7,44 \pm 0,97$ & $89,40 \pm 17,89$ & $97,37 \pm 6,47$ & $91,18 \pm 15,72$ \\
\hline & Superiores & $7,24 \pm 0,90$ & $85,67 \pm 23,34$ & $94,44 \pm 18,41$ & $89,89 \pm 17,63$ \\
\hline \multirow{3}{*}{ Estudios de la madre } & Básicos & $7,38 \pm 0,96$ & $84,78 \pm 22,95$ & $97,42 \pm 6,47$ & $86,29 \pm 21,45$ \\
\hline & Medios & $7,50 \pm 1,00$ & $88,48 \pm 20,02$ & $96,56 \pm 12,26$ & $91,23 \pm 15,95$ \\
\hline & Superiores & $7,19 \pm 0,93$ & $87,86 \pm 18,41$ & $97,01 \pm 7,77$ & $89,41 \pm 17,74$ \\
\hline \multirow{2}{*}{ Repetidor } & Sí & $7,167 \pm 0,88$ & $77,220 \pm 25,99$ & $90,998 \pm 16,62$ & $83,726 \pm 22,45$ \\
\hline & No & $7,481 \pm 0,99$ & $90,235 \pm 17,76$ & $98,893 \pm 4,52$ & $90,869 \pm 16,67$ \\
\hline
\end{tabular}

pia como primera opción tenían una mayor tasa de éxito $(p=0,03)$.

Para estudiar la relación con el curso, se realizó un análisis de varianza ANOVA, con el cual se observaba que la nota media y la tasa de rendimiento eran los indicadores que mostraban diferencias estadísticamente significativas entre los diferentes cursos académicos (Tabla V).
Para comprobar en qué sentido se producían estas diferencias se realizó la prueba post-hoc de Scheffé; en ella se comprobó que los alumnos de cuarto curso tenían mejor nota media que el resto de los cursos $(p=0,000)$, siendo esa diferencia mayor a medida que se desciende de curso. En cuanto a la tasa de éxito, existía una diferencia significativa entre los alumnos de cuarto y de primer curso $(p=$ 
0,028), es decir, los alumnos de cuarto aprobaban más que los alumnos de primero.

Los alumnos repetidores presentaban menor tasa de rendimiento $(p=0,007)$, tasa de intento $(p=0,007)$ y tasa de éxito $(p=0,040)$, pero no existían diferencias significativas en la nota media $(p=0,08)$.

Se relacionó a continuación el rendimiento con el nivel educativo de los padres. Tras la realización de la prueba ANOVA, pudo comprobarse que el nivel educativo de los padres no mostraba diferencias significativas con el rendimiento académico en cuanto a nota media $(p=0,59)$, tasa de rendimiento $(p=0,43)$, tasa de intento $(p=0,26)$ y tasa de éxito $(p=0,26)$.

Del mismo modo, se procedió al análisis de varianza ANOVA para el nivel educativo de las madres, pero tampoco se encontraron relaciones con las variables de nota media $(p=0,35)$, tasa de rendimiento $(p=0,60)$, tasa de intento $(p=0,89)$ y tasa de éxito $(p=0,34)$,

\section{Discusión}

En primer lugar, creemos que sería necesaria la unificación de criterios a la hora de medir el rendimiento académico, ya que en la mayoría de los casos se toma como medida del rendimiento académico solamente la nota media [12-14], mientras que en otros estudios se utilizan las tasas de rendimiento, intento y éxito $[1,15]$, e incluso en otros estudios se considera la tasa de abandono como predictor del rendimiento [16]. Esto dificulta la comparación entre los diferentes estudios.

Cabe destacar también que no sabemos si los estudiantes que no participaron en el estudio podrían sesgar los resultados porque, como investigadoras, no podemos tener acceso a su expediente académico personal sin previo consentimiento del alumnado, y tan sólo hemos obtenido las tasas de rendimiento de aquellos que accedieron a formar parte de la investigación firmando dicho consentimiento.

A continuación, haremos alusión a las variables sociodemográficas. Como hemos visto, la edad se relaciona inversamente con la nota media, la tasa de rendimiento y la tasa de éxito. Estos resultados coinciden con los de Padierna et al [17], que concluyen que la edad se relaciona inversamente con el rendimiento. Esto puede deberse a que a este factor se le sumen otras variables, ya que a mayor edad es habitual tener otras obligaciones $(p=0,010)$ y también es más frecuente que se compaginen los estudios con el trabajo $(p=0,000)$.

La relación entre el hecho de ser hombre o mujer y el rendimiento académico conseguido no es
Tabla II. Relación entre el rendimiento académico y la situación laboral.

\begin{tabular}{|c|c|c|c|c|c|c|}
\hline & $\begin{array}{l}\text { Trabaja en } \\
\text { la actualidad }\end{array}$ & $n$ & Media & $\begin{array}{l}\text { Desviación } \\
\text { estándar }\end{array}$ & $\begin{array}{l}\text { Error estándar } \\
\text { de la media }\end{array}$ & $\begin{array}{c}t \text { de } \\
\text { Student }\end{array}$ \\
\hline \multirow{2}{*}{ Nota media } & Sí & 31 & 7,24574 & 1,165317 & 0,209297 & \multirow{2}{*}{$p=0,396$} \\
\hline & No & 118 & 7,43963 & 0,927617 & 0,085394 & \\
\hline \multirow{2}{*}{$\begin{array}{l}\text { Tasa de } \\
\text { rendimiento }\end{array}$} & Sí & 31 & 77,1723 & 24,94450 & 4,48016 & \multirow{2}{*}{$p=0,003$} \\
\hline & No & 118 & 89,4213 & 18,95482 & 1,74493 & \\
\hline \multirow{2}{*}{ Tasa de intento } & Sí & 31 & 92,3787 & 17,94668 & 3,22332 & \multirow{2}{*}{$p=0,089$} \\
\hline & No & 118 & 98,1105 & 5,48392 & 0,50484 & \\
\hline \multirow{2}{*}{ Tasa de éxito } & Sí & 31 & 82,9842 & 20,83749 & 3,74252 & \multirow{2}{*}{$p=0,043$} \\
\hline & No & 118 & 90,5468 & 17,62215 & 1,62225 & \\
\hline
\end{tabular}

Tabla III. Relación entre el rendimiento académico y la vía de acceso a la universidad.

\begin{tabular}{|c|c|c|c|c|c|c|}
\hline & $\begin{array}{l}\text { Vía de ingreso a los } \\
\text { estudios actuales }\end{array}$ & $n$ & Media & $\begin{array}{l}\text { Desviación } \\
\text { estándar }\end{array}$ & $\begin{array}{l}\text { Error estándar } \\
\text { de la media }\end{array}$ & $\begin{array}{c}t \text { de } \\
\text { Student }\end{array}$ \\
\hline \multirow{2}{*}{ Nota media } & Selectividad & 114 & 7,64531 & 0,860107 & 0,080556 & \multirow{2}{*}{$p=0,000$} \\
\hline & Form. profesional & 34 & 6,64809 & 0,941019 & 0,161383 & \\
\hline \multirow{2}{*}{$\begin{array}{l}\text { Tasa de } \\
\text { rendimiento }\end{array}$} & Selectividad & 114 & 90,9227 & 18,24282 & 1,70860 & \multirow{2}{*}{$p=0,001$} \\
\hline & Form. profesional & 34 & 74,6135 & 23,73274 & 4,07013 & \\
\hline \multirow{2}{*}{ Tasa de intento } & Selectividad & 114 & 97,5278 & 9,74578 & 0,91278 & \multirow{2}{*}{$p=0,285$} \\
\hline & Form. profesional & 34 & 95,5179 & 8,98726 & 1,54130 & \\
\hline \multirow{2}{*}{ Tasa de éxito } & Selectividad & 114 & 92,8444 & 15,39774 & 1,44213 & \multirow{2}{*}{$p=0,000$} \\
\hline & Form. profesional & 34 & 76,8856 & 22,63713 & 3,88224 & \\
\hline
\end{tabular}

unánime en la bibliografía académica. Los resultados de nuestro estudio muestran que, aunque la tendencia es que las alumnas puntúen ligeramente más alto que los alumnos, en todos los aspectos, excepto en la tasa de éxito, estas diferencias no son significativas. Tales resultados concuerdan con el estudio de Rodríguez et al [18], quienes aseguran que no se puede afirmar una relación directa entre el rendimiento y el sexo. Sin embargo, hay estudios que otorgan a las mujeres una ligera tendencia al rendimiento superior que a los hombres, como Martínez et al [1], quienes en su estudio sí obtienen diferencias significativas a favor del sexo femenino. 
Tabla IV. Relación entre la nota de acceso a la titulación y los indicadores de rendimiento académico, por cursos.

\begin{tabular}{llcccc}
\hline & & $\begin{array}{c}\text { Nota } \\
\text { media }\end{array}$ & $\begin{array}{c}\text { Tasa de } \\
\text { rendimiento }\end{array}$ & $\begin{array}{c}\text { Tasa de } \\
\text { intento }\end{array}$ & $\begin{array}{c}\text { Tasa } \\
\text { de éxito }\end{array}$ \\
\hline $\begin{array}{l}\text { Primer } \\
\text { curso }\end{array}$ & Correlación de Pearson & 0,174 & 0,321 & 0,476 & 0,242 \\
\cline { 2 - 6 } & Significación (bilateral) & 0,303 & 0,053 & 0,003 & 0,148 \\
\hline $\begin{array}{l}\text { Segundo } \\
\text { curso }\end{array}$ & Correlación de Pearson & $-0,204$ & $-0,194$ & $-0,002$ & $-0,371$ \\
\hline $\begin{array}{l}\text { Sercer } \\
\text { curso }\end{array}$ & \begin{tabular}{l} 
Correlación de Pearson \\
\cline { 2 - 6 }
\end{tabular} & 0,427 & 0,096 & 0,060 & 0,114 \\
\hline $\begin{array}{l}\text { Significación (bilateral) } \\
\text { curso }\end{array}$ & Correlación de Pearson & 0,008 & 0,573 & 0,725 & 0,500 \\
\hline \begin{tabular}{l} 
Significación (bilateral) \\
\hline
\end{tabular} & 0,037 & 0,107 & 0,045 & 0,111 \\
\hline
\end{tabular}

Tabla V. Relación entre los indicadores de rendimiento académico y curso.

\begin{tabular}{|c|c|c|c|c|c|c|}
\hline & & $\begin{array}{l}\text { Suma de } \\
\text { cuadrados }\end{array}$ & $\begin{array}{c}\text { Grados } \\
\text { de libertad }\end{array}$ & $\begin{array}{c}\text { Media } \\
\text { cuadrática }\end{array}$ & $F$ & $\begin{array}{l}\text { Significa- } \\
\text { ción }(p)\end{array}$ \\
\hline & Intergrupos & 54,927 & 3 & 18,309 & & \\
\hline \multirow[t]{3}{*}{ Nota media } & Intragrupos & 87,711 & 147 & 0,597 & 30,685 & 0,000 \\
\hline & Total & 142,638 & 150 & & & \\
\hline & Intergrupos & $2.963,110$ & 3 & 987,703 & & \\
\hline \multirow[t]{3}{*}{$\begin{array}{l}\text { Tasa de } \\
\text { rendimiento }\end{array}$} & Intragrupos & $61.763,667$ & 147 & 420,161 & 2,351 & 0,075 \\
\hline & Total & $64.726,777$ & 150 & & & \\
\hline & Intergrupos & 188,964 & 3 & 62,988 & & \\
\hline \multirow{3}{*}{$\begin{array}{l}\text { Tasa de } \\
\text { intento }\end{array}$} & Intragrupos & $13.817,431$ & 147 & 93,996 & 0,670 & 0,572 \\
\hline & Total & $14.006,395$ & 150 & & & \\
\hline & Intergrupos & $3.601,806$ & 3 & $1.200,602$ & & \\
\hline \multirow{2}{*}{$\begin{array}{l}\text { Tasa } \\
\text { de éxito }\end{array}$} & Intragrupos & $47.401,485$ & 147 & 322,459 & 3,723 & 0,013 \\
\hline & Total & $51.003,292$ & 150 & & & \\
\hline
\end{tabular}

Albalate et al [12] aseguran que el hecho de que los alumnos residan en el área de la universidad debe suponer un ahorro de tiempo y esfuerzo, lo que podría influir de forma positiva en el rendimiento académico. Por otro lado, esto puede generar más tiempo libre y permitir una mayor integración en el am- biente universitario lo cual provocaría un efecto positivo [1]. Sin embargo, en su estudio, aseguran que el hecho de mudarse de la casa familiar a la universidad puede generar una sensación de desarraigo y alejamiento de la familia y producir un impacto negativo sobre el rendimiento, lo que podría justificar los resultados de nuestro estudio, puesto que vemos que los alumnos que no viven con su familia presentan una menor nota media $(p=0,06)$, rozando este aspecto el límite de significación del 5\%. Esto también podría relacionarse con otras variables, ya que hemos comprobado que existen diferencias significativas entre los alumnos que residen con su familia y los que no con otras obligaciones $(p=0,019)$, es decir, los alumnos que no residen con su familia correlacionan positivamente con otras obligaciones, lo que restaría tiempo para sus estudios.

Los alumnos que compaginan los estudios con el trabajo presentan una tasa de rendimiento y de éxito menores; estos alumnos aprueban menos y tardan más en terminar sus estudios, aunque existen investigaciones en las que se argumenta que el trabajo puede tener efectos positivos en el rendimiento académico, dado que favorece la transferencia de conocimiento relacionado con los temas de estudio. Aun cuando no se relacione con la carrera, esto posibilita la disciplina y refuerza el sentido de la responsabilidad en el desempeño académico [19], pero también constan otros estudios, como el de Caballero [20], en el que no se aprecian diferencias entre los alumnos que trabajan y los que no, en cuanto al rendimiento académico.

Los alumnos que tienen otras obligaciones además de los estudios no presentan diferencias significativas en el rendimiento académico, De Oliveira [21] afirma que hay estudiantes con hijos, por lo que sus obligaciones prioritarias son otras, o estudiantes que aunque no trabajan fuera de casa, se encargan de todas las tareas domésticas, y en ocasiones incluso de la crianza de sus hermanos más pequeños, sobre todo las mujeres (aunque cabe destacar que este estudio se ha realizado en una población en la cual la situación socioeconómica es de pobreza).

En cuanto a las variables socioeducativas podemos comprobar que la vía de ingreso parece ser una variable con gran influencia sobre el rendimiento académico, ya que los alumnos que provienen de formación profesional obtienen peores notas que sus compañeros que proceden de la selectividad; las tasas de rendimiento y de éxito también son menores en estos alumnos. Los resultados coinciden con el estudio [22] realizado sobre una muestra de alumnos de la Universidad Carlos III de Madrid, que destaca el fuerte efecto negativo del acceso a través de 
los cupos reservados a los titulados de formación profesional. Esta variable se considera incluso un factor de riesgo de abandono de la carrera: un estudio vinculó significativamente la vía de acceso de la formación profesional con la tasa de abandono [16]. Todo esto podría tener relación con la metodología de enseñanza-aprendizaje empleada; consideramos que la enseñanza en el bachillerato se parece más a la de la universidad que a la de los ciclos de formación profesional por su carácter teórico, sobre todo en los primeros años de la titulación. Además, habitualmente el alumnado que obtiene mejoras notas en educación secundaria obligatoria elige el camino de la selectividad frente al de los ciclos formativos para acceder a la universidad.

La nota de acceso presenta unas correlaciones diferentes en función del curso académico estudiado; así, los alumnos de primer curso que presentan una mayor nota de acceso se presentan más y aprueban más. En cuanto a los alumnos de segundo, los que presentan mayor nota de acceso aprueban los exámenes a los que se presentan en mayor medida. Los alumnos de tercero que tenían mayor nota de acceso a su ingreso obtienen una mayor nota media, y en los de cuarto curso no existían diferencias significativas. Estudios realizados en la enseñanza superior asociados al rendimiento académico enfatizan el valor de la nota media obtenida en las pruebas de admisión a la universidad como un predictor importante en el rendimiento académico, junto con los rendimientos académicos previos a la universidad [7]. Este estudio concuerda con nuestros resultados.

Díaz et al [3] concluyen que el ajuste con lo deseado y lo real es un excelente predictor del desempeño académico universitario, ya que son alumnos vocacionalmente satisfechos. La posibilidad de ubicarse en una carrera universitaria escogida en primera opción también representa un indicador valioso en el rendimiento académico del estudiante universitario. Bartual y Poblet [13] han observado que la ventaja a favor del aprobado de un estudiante que ha elegido la carrera en primera opción es superior a la correspondiente a los estudiantes que optaron por la carrera en segundo o tercer lugar. Sin embargo, en nuestro estudio, los resultados no concuerdan con estas investigaciones, ya que los alumnos que no habían elegido fisioterapia como primera opción presentaban una mayor tasa de éxito. Hemos comprobado si existía relación entre esta variable (elección de la titulación como primera opción) y la nota de acceso y evidenciamos -no siendo estadísticamente significativo- que el alumnado que escogió fisioterapia como primera opción obtenía me- jor nota de acceso. Esto indica que quienes tienen notas más bajas escogieron otras carreras antes que fisioterapia, como podría ser medicina. En nuestro centro se cuenta con la figura del tutor curricular y en estas tutorías es frecuente encontrarse con alumnos que han elegido como primera opción otra carrera por consejo de sus padres, aunque realmente sus preferencias eran otras. Esto puede deberse, como exponen Torres y Rodríguez [14], a que existe cierto consenso social acerca de las carreras 'notables' y otras que no lo son tanto; por ejemplo, hay cierta propensión a sobrestimar carreras como medicina, derecho, ingeniería y administración. Si esta hipótesis fuese cierta, cuando acceden a fisioterapia como segunda opción, se eliminaría todo el contexto social y sólo primarían los intereses y gustos de este alumno que quizás sean más elevados por la fisioterapia que los que la eligieron como primera opción.

Los alumnos de cuarto curso obtienen una mayor nota media que los alumnos del resto de los cursos, siendo esa diferencia más significativa a medida que descendemos de curso. En cuanto a la tasa de éxito, existe una diferencia significativa entre los alumnos de cuarto y de primero. Los alumnos de primer curso obtienen peores notas que los alumnos de cuarto y tienen una menor probabilidad de aprobar. Este aspecto podría relacionarse con la adquisición de estrategias de aprendizaje que el alumno adquiere a medida que avanzan los cursos académicos [23]. Para comprobar esto, deberíamos realizar un análisis longitudinal del mismo grupo de alumnos para analizar los cambios en sus notas a lo largo de toda la titulación, evaluando si se aumenta la nota media a medida que progresa en los cursos. No contamos con estos datos, ya que efectuamos un estudio de carácter transversal y no longitudinal.

El nivel de estudios del padre y de la madre no se relaciona con el rendimiento académico, pero numerosos estudios muestran que, en general, a mayor cantidad de años de educación de los padres, mayor es el tiempo dedicado al estudiante y mayor es la calidad de la supervisión a éste [1]. Majluf [24] asegura que los padres de bajo nivel socioeducativo utilizan estrategias poco efectivas para enseñar a sus hijos -aunque valoren la educación y deseen que ellos tengan un buen rendimiento en la escuela- e interactúan escasamente con ellos en actividades relacionadas con estrategias de aprendizaje.

Los alumnos repetidores presentan menores tasas de rendimiento, intento y éxito, pero no existen diferencias significativas en la nota media. Según Albalate et al [12], y coincidiendo con nuestros resultados, el hecho de ser repetidor no parece afectar a los resultados académicos de la asignatura. Martín 
et al [25] justifican que ciertos estudiantes tardan más en superar los estudios porque realizan un aprendizaje de mayor calidad y complejidad cognitiva, y por ello no se vería afectada su nota media.

La principal limitación de este estudio es su carácter transversal y que se ha desarrollado en un solo curso académico y en una única titulación. Para poder obtener la validez externa de estos resultados sería necesario profundizar en la investigación ampliándola a otras titulaciones y llevándola a cabo en posteriores cursos académicos.

En conclusión, las variables sociodemográficas que poseen mayor influencia negativa sobre el rendimiento académico son ser mayor de 25 años y compaginar estudios y trabajo, siendo la primera la única variable sociodemográfica que influye negativamente en la nota media.

Las variables socioeducativas que inciden negativamente sobre el rendimiento académico de los estudiantes de fisioterapia son que la vía de acceso haya sido formación profesional, la nota de acceso, que la primera opción de matrícula haya sido fisioterapia, el cursar el primer año de carrera, y ser repetidor. De las variables expuestas, el haber accedido desde formación profesional y cursar primero son las variables que afectan a la nota media del alumnado.

\section{Bibliografía}

1. Martínez C, Rúa A, Redondo R, Fabra ME, Núñez A, Martín MJ. Influencia del nivel educativo de los padres en el rendimiento académico de los estudiantes de ADE. Un enfoque de género. AEDE 2010; 5: 1273-94.

2. Pizarro R. Rasgos y actitudes del profesor efectivo. Santiago de Chile: Pontificia Universidad Católica; 1985.

3. Díaz M, Peio A, Arias J, Escudero T, Rodríguez S, Vidal GJ. Evaluación del rendimiento académico en la enseñanza superior. Comparación de resultados entre alumnos procedentes de la LOGSE y del COU. RIE 2002; 2: 357-83.

4. Morales AL. El entorno familiar y el rendimiento escolar. Sevilla: Consejería de Educación y Ciencia; 1999.

5. Agencia Nacional de Evaluación de la Calidad y Acreditación (ANECA). Guía de apoyo para la elaboración de memoria para la solicitud de verificación de títulos oficiales (grado y máster). 2012. URL: http://www.aneca.es/Programas/ VERIFICA/Verificación-de-Grado-y-Master/Protocolos -de-evaluacion-y-documentos-de-ayuda.

6. Caso J, Hernández L. Variables que inciden en el rendimiento académico de adolescentes mexicanos. Revista Latinoamericana de Psicología 2007; 39: 487-501.
7. Garbanzo GM. Factores asociados al rendimiento académico en estudiantes universitarios, una reflexión desde la calidad de la educación superior pública. Rev Educ 2007; 31: 43-63.

8. Marchesi A. Un sistema de indicadores de desigualdad educativa. RIE 2000; 23: 135-64.

9. Hess RD, Holloway SD. Family and school as educational institutions. Review of child development research VII. Chicago: University of Chicago Press; 1984.

10. Tejedor FJ, García-Valcárcel A. Causas del bajo rendimiento del estudiante universitario (en opinión de los profesores y alumnos). Propuestas de mejora en el marco EEES. Rev Educ 2007; 342: 443-73.

11. Ministerio de la Presidencia. Real Decreto $1892 / 2008$ por el que se regulan las condiciones para el acceso a las enseñanzas universitarias oficiales de grado y los procedimientos de admisión a las universidades públicas españolas. Boletín Oficial del Estado, n. ${ }^{\circ} 283$, de 24 de noviembre de 2008.

12. Albalate D, Fageda X, Perdiguero J. Éxito académico, características personales y proceso de Bolonia: una aplicación econométrica. RIDU 2011; 3: 11-25.

13. Bartual T, Poblet MC. Determinantes del rendimiento académico en estudiantes universitarios de primer año de economía. REFIEDU 2009; 2: 172-81.

14. Torres LE, Rodríguez NY. Rendimiento académico y contexto familiar en estudiantes universitarios. Enseñ Invest Psicol 2006; 11: $255-70$.

15. Casuso MJ. Estudio del estrés, engagement y rendimiento académico en estudiantes universitarios de ciencias de la salud. Málaga: Universidad de Málaga; 2011.

16. Corominas E. La transición a los estudios universitarios. Abandono o cambio en el primer año de universidad. Rev Investig Educ 2001; 19: 127-51.

17. Padierna JL, Oseguera J, Gudiño N. Factores socioacadémicos, estilo de aprendizaje, nivel intelectual y su relación con el rendimiento académico previo de médicos internos de pregrado. Educ Med 2009; 12: 91-102.

18. Rodríguez S, Fita E, Torrado M. El rendimiento académico en la transición secundaria-universidad. Rev Educ 2004; 334: 391-414.

19. Holland A, Andre T. Participation in extracurricular activities in secondary school: what is know, what needs to be know? RER 1987; 57: 4347-466.

20. Caballero CC. Burnout, engagement y rendimiento académico entre estudiantes que trabajan y aquellos que no trabajan. Psicogente 2006; 9: 11-27.

21. De Oliveira O. Transformaciones socioeconómicas, familia y condición femenina. Familia, género y pobreza. México: Miguel Ángel Porrúa; 2000.

22. García MM, San Segundo MJ. El rendimiento académico en el primer curso universitario. X Jornadas de la AEDE; 2001.

23. Lassibille G, Navarro L. El valor del tiempo en la universidad. Málaga: Universidad de Málaga; 1990.

24. Majluf A. Marginalidad, inteligencia y rendimiento escolar. Lima: Brandon Enterprise; 1993.

25. Martín E, García LA, Torbay A, Rodríguez T. Estrategias de aprendizaje y rendimiento académico en estudiantes universitarios. International Journal of Psychology and Psychological Therapy 2008; 8: 401-12. 